\title{
RELATO Y LENGUAJE. HACIA UNA BASE PARA LA CRÍTICA DEL CONSUMO
}

\author{
Gustavo Garduño Oropeza ${ }^{1}$ y Robert Stingl ${ }^{2}$
}

\section{Introducción}

Algunas de las consecuencias que implicó la consolidación del mercado tienen que ver con lo que Baudrillard denominó el "surgimiento de una nueva ecología" y guardan estrecha relación con la forma por la que se modifica la interacción entre las necesidades del ser humano en la modernidad y las diferentes vías de satisfacción que el mercado engendra.

Entre dichas consecuencias encontramos las siguientes:

a) La transformación de la naturaleza de las mercancías, las cuales pasan de ser una respuesta a necesidades inmediatas para convertirse en meros intermediarios entre el sujeto y nuevos procesos de producción realizados a una escala diferente. Esta última destinada al desarrollo y comercialización de nuevas formas de mercancía. Se trata de la fase de producción en la que se da la transformación del objeto producido desde su dimensión material hacia la simbólica.

Fue en esta fase cuando se lleva a cabo una inversión de la relación que las mercancías guardan con las necesidades pues estas últimas dejan de ser un fin para convertirse en un medio mientras la propia mercancía deja de ser medio para volverse fin.

b) La transformación de la relación del sujeto con la mercancía altera tanto la forma de trabajo como el entorno cultural para adecuarse a condiciones o exigencias impuestas

\footnotetext{
${ }^{1}$ Profesor-Investigador de la Facultad de Ciencias Políticas y Sociales de la Universidad Autónoma del Estado de México, Doctor en Humanidades, miembro del Sistema Nacional de Investigadores I e integrante del cuerpo académico Difusión y divulgación de la Ciencia en dicha institución superior. Tel. Celular: 7223572126, Dirección Electrónica: gustavoa.gardunoo@gmail.com. Dirección Física: Cerro de Coatepec, s/n Facultad de Ciencias Políticas y Sociales. Ciudad Universitaria, Toluca, Estado de México 50100.

2 Profesor-Investigador de las Facultades de Ciencias Políticas y Sociales y Lenguas de la Universidad Autónoma del Estado de México, Maestro en filosofía y Titular de seminario de investigación en Crítica de lenguaje. Actualmente estudia el doctorado en Estudios Latinoamericanos en la Facultad de humanidades de la misma casa de estudios. Teléfono: 72233910252. E-mail: robert.stingl@univie.ac._Dirección Física: Cerro de Coatepec, s/n Facultad de Ciencias Políticas y Sociales. Ciudad Universitaria, Toluca, Estado de México 50100.
} 
por un esquema de necesidades construidas. La idea de consumidor aparece cuando el sujeto comienza a operar sujeto a una idea de desarrollo o progreso.

Con el advenimiento de la modernidad y particularmente desde la coyuntura específica del neoliberalismo en la que el dinero desplaza a la convivencia, a la socialización, tanto el reconocimiento como la trascendencia se convierten en motores de una producción ya no dirigida a la satisfacción sino a la acumulación.

c) Y es precisamente cuando la idea de "desarrollo"3 se materializa -a partir de dicha acumulación- que el consumo se convierte en el único mecanismo por el que un sujeto puede interactuar con su entorno; un entorno perfectamente apto para ponderarlo a través de sus consumos y vincularlo con diferentes tipologías sociales. Esta última idea podría referir al mapa de esa "nueva ecología” en donde las relaciones ya no son entre especies, sino entre consumidores, mercancías y relatos.

\section{El relato y el desarrollo}

\footnotetext{
${ }^{3}$ La idea de desarrollo aparece aquí en su sentido semántico más amplio: como la de un proceso permanente de acoplamiento a parámetros o ideas emanadas de centros de poder económico y político encargados de calificar gobiernos, instituciones y organizaciones.
} 
"Los animales -y los hombres entre ellos- no perciben las situaciones y las cosas con todas sus determinaciones materiales, sino sólo 'signos' de ellas" (Ibáñez, J. 2012).

Son precisamente esos signos de los que nos habla Jesús Ibáñez los que articulan la nueva ecología. Es en virtud de ellos que la sociedad de consumo se erige al modo de un inmenso compendio de instrucciones o relatos que vinculan a la mercancía y al consumidor a través de redes de sentido en las que la necesidad y la satisfacción se reconfiguran permanentemente. Consideremos para ilustrar este proceso el caso del software especializado de uso cotidiano o lo que tradicionalmente llamamos “Apps". Una aplicación no es sino una mercancía que emerge de una necesidad construida a partir de una indeterminación técnica, un vacío semántico. Pero: ¿de dónde parte?... de otra mercancía y se trata de un derivado de tercer o cuarto orden proyectado en función de las propiedades o condiciones de un producto anterior para el que la comunicación entre personas -a todas luces- no es su principal cometido: El teléfono celular.

Al poder proyectar o capturar información de orden diversa, la aplicación elimina un vacío de sentido (u opción abierta) y hace significativo el uso del dispositivo: El celular nos permite, entonces, hacer proyecciones de temperatura, localizar cafés o bares, identificar a otros usuarios cercanos y hasta capturar monstruos virtuales en espacios reales... Todo ello parte de una configuración de sentido, de un relato que nos adentra en lo probable dejándonos en claro que ésta será posible sí y solo sí vinculamos mercancías con nuevas mercancías y ámbitos de uso.

Ibáñez (2012:23) lo expone así: “Jugando con diferencias meramente formales se abre [la posibilidad a] una serie infinita de productos diferentes". Y esa es precisamente lo que

implica la lógica del desarrollo: un concepto tramposo que depende, para su comprensión, de indicadores o referencias. Un punto de partida para la posibilidad. 
Vincular al relato con el desarrollo implica, entonces, un proceso similar al que se aplica al generar discursos políticos que sirvan tanto a críticos como a apologetas de un determinado gobierno: Sobre un cúmulo características ambiguas (democracia) se eligen ciertas formalizaciones a partir de las cuales se construyen escenarios relatados posibles. Para Jesús Ibáñez podría implicar la lógica de la red que se forma en torno a un punto de quiebre sobre el que se articula un orden que nos cubre de las contingencias: El propio mercado. Una red que operaría algo así como:

....antes de poder regular la circulación por la red ha habido que construir la red: con las variaciones sobre el prefijo 'teg' se construye términos que denotan [...] techo, tela, tejido, toga, texto... es decir todo lo que protege. (Ibáñez, J. 2012: 115).

La cosa será preguntarnos sobre esa protección, sus alcances y las formas por las que ésta no puede convertirse en un riesgo mayor al de la ambigüedad que pretende catalizar. Tenemos entonces una triada:

\section{Figura 1 (Triada Formalización, Ambigüedad, Posibilidad=Sentido).}

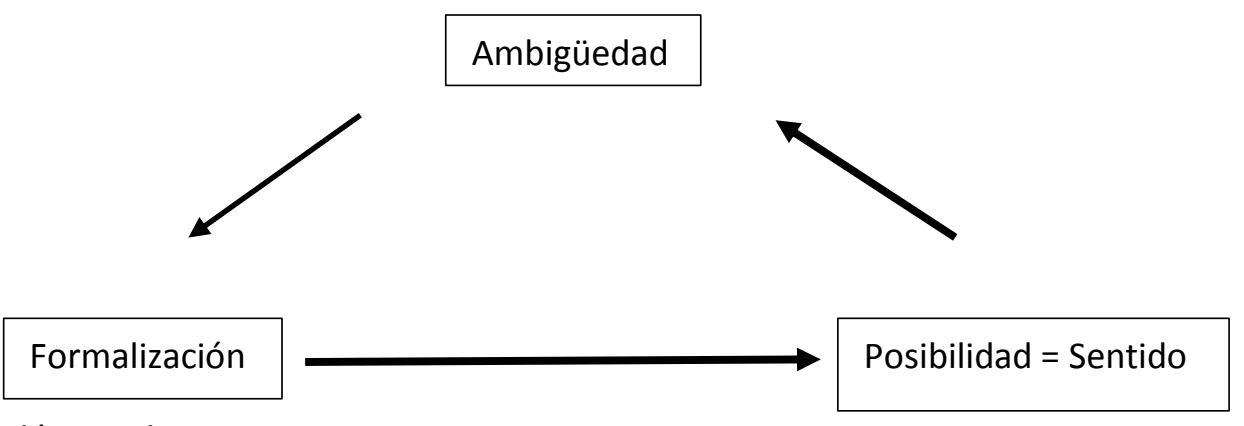

Fuente: Elaboración propia.

A través de la relación entre las distintas casillas (fig. 1) es posible comprender cómo, a partir de la ambigüedad, un discurso se teje eligiendo una serie de características o condiciones que, al formalizarse, abren la posibilidad a comprender la naturaleza de una necesidad emergente. Ésta, a su vez, abrirá en un nuevo proceso otra puerta a la indeterminación y, así, 
se cae en una espiral de desarrollo basado en un ajuste permanente entre necesidades y mercancías. Mediante este ejercicio se puede llegar a comprender la forma por la cual se llegó a la primera gran consecuencia de la consolidación del mercado: la ecología del consumo desplazó la naturaleza de la mercancía de ser medio a ser fin.

Para la segunda consecuencia se puede inferir un cambio sustancial en el papel que adquiere el sujeto al perder contacto directo con necesidades de primer orden. Bajo condiciones de un trabajo emergente en el que se le remunera con dinero y no mediante la obtención de productos, el hombre deviene en consumidor y sus necesidades quedan referidas por la oferta. La ambigüedad queda catalizada por el repertorio mismo de mercancías que se le ofrecen para cambiar por su salario y, por lo tanto, sus formalizaciones dependerán del rol que él mismo se asigne frente a la ambigüedad de éstas. El consumidor es el sujeto social referido por el relato de la mercancía y encuentra su idea de desarrollo en el acoplamiento o ruptura con determinadas formalizaciones.

Finalmente, el desarrollo aparece como un término análogo al consumo. La posibilidad de acumular mercancías permitirá definir roles sociales entre grupos sociales cada vez más complejos, heterogéneos y dinámicos que, no obstante, mantienen en común el criterio del capital como única medida.

El desarrollo, explicado según la lógica de la figura 1, podría aparecer como una espiral que se prolonga indefinidamente en la que cada curva, cada pliegue, cada recurrencia estén dados por la transformación de cada posibilidad o sentido adquirido en un nuevo marco de

ambigüedad o indeterminación. Se trata del tejido de la red. El punto que se prolonga haciendo del contexto un sistema y de cada acción una forma de integración. 
La idea de mercancía hasta este punto es la de una materialidad que cuenta, en sí misma, con las instrucciones para acoplarse (y acoplar a quien la consume, ostenta y sustituye) en una lógica e interacciones externa (desarrollo).

\section{Figura 2. (Ambigüedad, Formalización, Desarrollo, Posibilidad Sentido)}

\section{Ambigüedad}

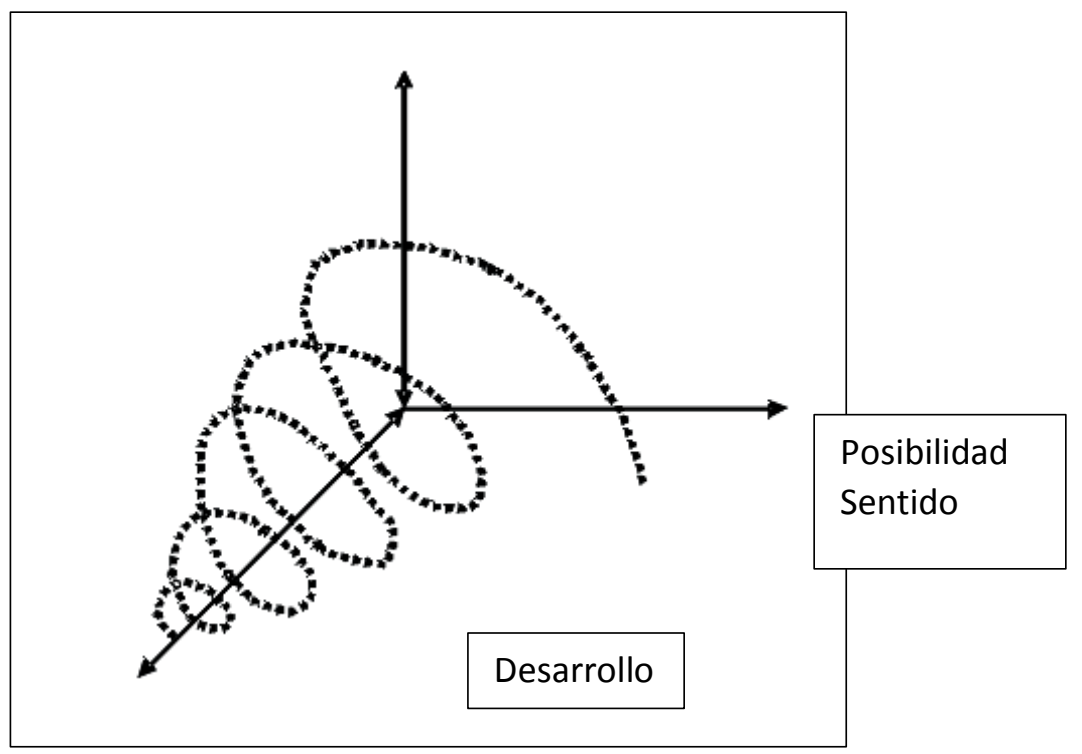

Formalización

Fuente: Elaboración propia.

Este vínculo entre materialidad y significado será abordado en el siguiente punto y consideraremos hablar de relatos para referir la parte simbólica de la mercancía en tanto ésta

aparece referida en conjuntos de instrucciones colaterales llamados mercadotecnia, publicidad o propaganda ${ }^{4}$.

\section{La producción de relatos}

${ }^{4}$ Obviaremos la definición de cada uno de estos conceptos en tanto resultan colaterales a nuestra argumentación. 
"En el campo del consumo, la conexión física se acopla a la conexión simbólica" (Ibáñez, 2012: 31)

Desde que el trabajo dejó de ser una actividad doméstica, el espacio cotidiano dejó de estar determinado por las prácticas y definió en función de representaciones. Hasta antes de la consolidación del mercado como fuente de suministros para satisfacer necesidades diarias, la configuración de los espacios domésticos estaba dada por los trabajos y la geografía de insumos y herramientas muchas veces producidos internamente. Hablamos de instrumentos y materiales para el campo y el cuidado del ganado; de instrumentos y materiales para la conservación y preparación de alimentos y de instrumentos y materiales para la subsistencia de individuos (ropa, calzado, alcoba). Los únicos recursos simbólicos presentes en esas dinámicas estaban profundamente ligados al culto y a la participación en ciertos ritos de la vida comunitaria; se trataba de auténticas excepciones. Lo que distinguía -entonces- la configuración de la vida cotidiana en este contexto era la presencia, posición y jerarquía de insumos que satisfacían necesidades inmediatas; insumos que no tenían más sentido que su propia función y cuyo uso se ligaba al dominio en su manejo y una cierta inercia cultural (actividades propias de varones, de mujeres y de niños).

Cuando el trabajo se desplaza de la casa a la fábrica y las familias rompen con aquella división del trabajo tradicional para convertirse en asalariados, la configuración de lo cotidiano muta a su vez y nos obliga a replantear el papel que jugarán -entonces- los objetos en las nuevas formas de vida familiar.

Haciendo a un lado por fines prácticos los extremos degradantes a los que la industrialización primaria llevó a los trabajadores (primera revolución industrial), consideraremos que la mutación en la configuración del cotidiano parte de la conversión de lo que podríamos llamar "familias productoras" en "familias consumidoras" que, una vez fuera de sus espacios de trabajo, se enfrentaron al replanteamiento de lo que era y lo que tenía que ser una casa. 
En esta fase [segunda revolución industrial], las actividades de ocio y consumo eran, aún, relativamente propias: los trabajadores conservaban en sus casas burbujas de espacio y de tiempo que llenar con su actividad [pero no había ya actividad más allá de la nave industrial. El desafío que se abría para ellos comenzó a ser aprender a vivir para sus pocas posesiones y fue así que se llegó al punto en el que]...el capitalismo de consumo nos despoja -regularizándolasde nuestras actividades de ocio y consumo: crea necesidades que se acoplen a los productos que está interesado en vender, transformando -mediante la pedagogía y la demagogia- nuestras actividades de ocio y consumo para que se acoplen a sus productos. (Ibáñez, 2012: 47. Los corchetes son nuestros).

Y en ese acoplamiento fue que apareció la necesidad de relatos, de mecanismos de significación que permitieran a la clase trabajadora forjar un nuevo hábitat y nuevas relaciones cotidianas en la metrópoli a través del consumo de los productos que ellos mismos contribuían a ensamblar en la línea de producción.

La aparición del ocio fue importante en esta gesta y para ello se le tuvo que asociar con la idea de que el aprovechamiento de tal espacio improductivo podía ser la puerta de entrada a una condición de permanente desarrollo personal. El aprovechamiento del tiempo libre en el perfeccionamiento de la actividad, en la preparación y en el manejo de nuevas y más complejas formas de información estaba dirigido a que el trabajador pudiese ver al trabajo como una especie de competencia en la que el premio consistía en escalar socialmente. El trabajador tenía que ser consciente de que su esfuerzo podía llevarlo a "ser más" en la medida en la que produjese más y aprovechase los momentos libres para competir con sus pares.

Más adelante esta competencia permitió que el terreno de lo cotidiano, sus espacios de intimidad y el tiempo libre fuesen colonizados por la idea de desarrollo. Hoy por hoy no se puede hablar de mercado, consumo y consumidores sin la previa existencia de esta competencia enfermiza solo justificada por la búsqueda de un avance permanente que nos reposicione frente a los demás. 
El desarrollo constituye hoy día la piedra angular sobre la que se erige el edificio moderno. Se trata de una especie de sustituto de la divinidad que generó tanto su propia mitología como los rituales que la materializarían y entre ellos estuvo el brindar, a través del dinero, la posibilidad de hallar un nivel en la estructura social. La idea de llegar a un punto, la ubicuidad de dicho punto y la serie de prácticas por las que se pretende alcanzarlo prefiguran al consumidor como la figura más emblemática con la cual podemos referir al sujeto moderno. Y es que éste ha cedido las prácticas unidas a su determinación por nacimiento a las representaciones alcanzadas a través de su participación en el consumo. Si continuó inmerso en dinámicas laborales, éstas ya no supusieron una imposición sino una elección de las opciones que mejor le permitiesen escalar una cuesta social cuya configuración resultó ser eminentemente paramétrica: ingreso, educación, ámbito educativo, grados obtenidos, jerarquía laboral, grupos o instituciones que lo legitiman, nivel crediticio, bienes acumulados, redes de relación, presencia mediática, etc....

Pero más allá de estos criterios, la relación que el sujeto guarda con sus consumos abre hoy día una puerta más para la construcción de su identidad. Baste preguntarse: ¿Qué tanto me define como persona el auto que manejo, la ropa que uso, la plataforma computacional de mi elección (Mac o Windows), la bebida energética que me acompaña al gimnasio... el gimnasio en sí?

Al parecer, más allá de los vínculos institucionales que nos dan una cualidad social paramétrica, hay vínculos de consumo que nos proporcionan una alternativa de identidad y

ésta aparece como consecuencia de complejos sistemas de relato a los que llamamos "marketing": las prácticas que tienen como fin adaptar los productos o servicios a esquemas de sentido tanto asegurados como emergentes.

\section{a). El relato asegurado.}

Parte de la configuración de sentido asignada a un producto o servicio con base en elementos de fácil reconocimiento cultural por estar estabilizados (como estereotipos, prejuicios u opiniones de autoridad). 


\section{b). El relato emergente.}

Parte de la configuración de sentido asignada a un producto o servicio con base en criterios de innovación, exclusividad o, incluso, una novedad no identificable, escandalosa o contraria a moral, sistemas de creencia e inercias históricas socialmente reconocidas.

La correcta mezcla entre ambas configuraciones es la que se considera base del éxito o fracaso de una campaña (finalmente el relato con el que se da sentido a la mercancía).

Resulta importante para quien hace marketing (particularmente si su actividad se centra en el ámbito creativo, diseño de empaque o placement) no escatimar en la adquisición de habilidades narrativas dado que su trabajo va a ser producirlas con igual o mayor frecuencia a aquella esperada en un literato o un ensayista. Recordemos que el mercadólogo no solo opera con la palabra sino con lenguajes yuxtapuestos ${ }^{5}$ con los que se buscará generar una experiencia a la vez comprensible, pero también polémica, deseable y concreta susceptible de representación en diferentes escenarios comerciales. Esta última es quizá la fase que constituirá el puente entre un público y un producto o servicio. (Garduño, UAEM. 2015: Pre-print).

\section{Relato y paradojas del mundo de sentido comercial}

La trascendencia que se presentaba al individuo y la consistencia que éste tenía frente a las formas de vida pre-modernas no pudo ser compensada por la idea de un desarrollo permanente. La acumulación por la acumulación misma, el capital como nexo y la ubicuidad de un punto para la trascendencia limitaron los alcances de esferas filosóficas como la ética, la estética y la axiología, reduciéndolas a las posiciones del sujeto frente a la producción. La era del desasosiego inicia cuando el consumidor (masificado) reconoce las recompensas en el mero mantenimiento y en la actualización permanente de sus propios consumos (que, por

\footnotetext{
${ }^{5}$ Audiovisuales, escénicos, multimedia.
} 
cierto, son los de todos los demás). Por ello, bajo el guion presentado por la industria cultural $^{6}$, las opciones que se presentan como alternativa a una forma de vida de mercado se reducen a dos:

a) El acceso a estilos de vida alternativos basados un desarrollo local (esquemas que posiblemente sólo se ubiquen en comunidades como los menonitas o grupos intelectuales de corte prerrafaelista).

Un estilo de vida hipotético que podría resultar ser consecuencia de la insostenibilidad del esquema de producción/consumo masificados y sus consecuencias.

b) La expansión irrestricta de estilos de vida propios del actual modelo de desarrollo global del capital y del mercado, así como el consecuente advenimiento de políticas autoritarias que busquen contrarrestar los efectos perversos del mismo: políticas que privilegien acceso, uso y disfrute de acuerdo con el único criterio posible, el del rol económico.

El estudio de ciertas consecuencias perversas de esta expansión ya se había realizado desde la década de los 70’s por el filósofo austriaco Iván Illich, quien desarrolló conceptos como “contra-productividad” para señalar las paradojas de la producción irrestricta de tecnología y servicios ${ }^{7}$. Para este autor, el incremento de los consumos de bienes naturales y energía para la producción se acelerará (por la incapacidad del consumidor de vivir fuera de los esquemas inducidos por la producción y modelos de satisfacción) mientras se presenta un efecto contrario en los indicadores de bienestar que se verán seriamente reducidos gracias a la congestión vial que inhibe la movilidad, al desarrollo de nuevas enfermedades que emerjan de los propios remedios de la medicina, al cambio climático -producido por la desmedida

\footnotetext{
${ }^{6} \mathrm{Cf}$. Horkheimer y Adorno, 1994. El concepto hace referencia no solo a los alcances que la mercadotecnia y la publicidad tienen, sino a la forma como éstas integran en su discurso imágenes socialmente interiorizadas gracias a la acción conjunta de medios y prácticas de sentido en el mundo moderno: cine, televisión, radio, nuevas tecnologías. Pero también ritos de consumo, formas y estilos de vida y hasta producción artística.

7 "Más allá de ciertos límites, la producción de servicios hará a la cultura más daños de los que la producción de mercancías causó a la naturaleza” (Illich, 1972).
} 
intervención del hombre- que reduzca la esperanza de vida, sustitución de insumos, abaratamiento de la educación, etc. y a una emergente reducción de los niveles de producción.

Acotar las contradicciones del desarrollo parece hoy ser una de las nuevas tareas de un marketing que busca generar relatos en torno a nuevas formas de vida, más amigables con el ambiente y más consistentes con la idea de una vida comunitaria y de aprovechamiento no solo de los recursos autogenerados sino del reciclamiento, la innovación en servicios y una nueva idea de "calidad" más basada en la naturaleza de los insumos que en la consistencia y homogeneización del proceso por los que se convierten en mercancías.

¿Qué tendría que modificarse? O, peor aún, ¿cómo? Tal es el papel de la crítica en las sociedades contemporáneas: brindar la posibilidad de colocarnos como antítesis ante nuestras aseveraciones cotidianas mediante la confrontación de sus consecuencias. ¿Democracias? ¿Desarrollo? ¿Conectividad? ¿Comunicaciones? ¿Bienestar? ¿Felicidad?

Los conceptos guía aparecen plagados de contradicciones y quizá en la ubicación de las mismas y el reconocimiento del antónimo sea donde podamos ubicar el punto de partida.

\section{Crítica al consumo a través de la simplificación (de lo masivo a lo local).}

Desde Tocqueville la idea de "masa" implica una doble lectura: por un lado, el descubrimiento de la barbarie de la sociedad, por otro, la posibilidad de adecuarla a diferentes fines como la ontología amorfa que es.

Producto de una sociedad que cambió un cotidiano doméstico (determinado por las labores productivas tendientes a satisfacer necesidades precisas) por una vida de consumo (tendiente a llenar los espacios del ocio hacia el desarrollo), las masas quedaron uniformadas por su rol frente al capital. Éste fue el que determinó la barbarie de la posibilidad (poca o mucha) frente a la oferta de mercado y, a la vez, abrió la puerta a la manipulación colectiva. La masa es el 
objeto de toda industria cultural y sobre ella recae el peso de los relatos que no son otra cosa sino la configuración misma de las formas que esa materia proteica debe de tomar.

Hoy por hoy, la masa tiene la dimensión del mercado y se ajusta al alcance que éste tiene. A través de los medios, las masas han adquirido proporciones globales y hoy es prácticamente obvio que los subproductos de la industria cultural son recursivos en diferentes latitudes e identificables por tendencias y consumos similares ${ }^{8}$. No obstante, es necesario reconocer que las filiaciones que se repiten a lo largo y ancho del planeta generan consumos diferenciados que rompen la estandarización o uniformidad que se supondría en una masa. De allí, la idea de consumos parciales puede redefinirse -mediante el discurso adecuado y su instrumentación en los grandes relatos de la IC-como consumos locales que eventualmente rompan la idea de una sociedad vinculada a la producción masiva y la lleven a la posibilidad de una producción sí estandarizada pero local mucho más simple y cercana a la función.

Se puede, del párrafo anterior, aludir a la ecuación manejada en el primer capítulo: la relación entre formalización y posibilidad como base para una nueva producción pero de alcance más acotado que, lejos de generar ambigüedad o indeterminación, asigne un sentido local, familiar o comunitario a los consumos. La idea de marca deja de ser asunto de logotipos y se convierte en una especie de denominación de origen... Esto ya funciona con consumos selectos como el de los alimentos, las bebidas y ciertas formas de arreglo personal que impactan a sectores específicos de la población.

La actual idea de masa debe desecharse pues liga al sujeto a una sola imagen del desarrollo: aquella con la cual se pretende moldearla. Quizá sea más preciso, sí, hablar de consumos y éstos estar ligados a una nueva concepción del desarrollo. Una más simple, más directa que emplace al sujeto, al consumidor a definirse no en función de grandes tendencias sublimadas, sino de opciones de futuro. Ya no se trata de trascendencia, sino de pertinencia.

\footnotetext{
${ }^{8}$ Grupos o "tribus" urbanas: hípsters, indies, grunges, punks, darkies, geeks, frikis, weeaboos...
} 


\section{Crítica a la mercadotecnia (de lo masivo a lo local)}

La realización de un análisis que exponga los mecanismos de sentido que vinculan al objeto con los sujetos, es decir: la subjetivación de la mercancía entendida como una forma de sublimación que, fuera de sí misma, carece de todo sentido.

Dicho análisis podría partir de la naturaleza de la formalización, es decir, de los enunciados por los cuales se construye un determinado sentido al consumo y tendría que centrarse en las campañas. A continuación enumeramos algunas de las opciones (muchas de ellas románticas e ideales) del camino al que podría conducir una crítica a las construcciones del lenguaje que pretenden desambiguar el alcance de un producto o servicio en aras de un bien mayor o de una funcionalidad más clara.

- Redefinición de los parámetros de desarrollo. Identificación de valores nuevos que, más allá de la definición de la trascendencia personal, busquen la permanencia (ecológica, folclórica, axiológica, tradicional) de la colectividad.

- Jerarquización de la trascendencia a través del discurso comercial: Privilegiar lo local frente a lo global, universal o masivo.

- Búsqueda permanente de la simplificación de los consumos a través de la vuelta a las funciones. Racionalidad vs emotividad al momento de la compra.

- Reconocimiento y tratamiento discursivo para la promoción de conceptos emergentes que impliquen a las consecuencias del consumo: PE "contraproductividad" (Illich), “solucionismo" (Morozov), “simplificación” (Maeda), “provincialización” (Sánchez), “retorno de saberes” (Foucault), “comunitarismo” (Harvey) , “vida cotidiana” (Certeau), “distopía y utopía” (Mumford), etc.

- Limitación del alcance discursivo de las campañas mediante el reconocimiento de los límites del producto promovido. Des-sublimación. 
- Aprovechamiento de las nuevas tecnologías y la llamada "democratización de las comunicaciones" para generar y promover mayor información sobre naturaleza, función e impacto de ciertos consumos. Particularmente de aquellos que enfrentan la evidencia de su impacto social y ambiental.

La necesidad de mercadotecnias alternativas es clave en la construcción de nuevos horizontes discursivos que modifiquen ya el afán desarrollista o los elementos a través del cual éste sería ponderado. No referimos a la puesta en marcha de un esquema de valor que se aleje de lo eminentemente cuantificable y busque lo sustentable. Illich lo llamaría:

Género vernáculo, es decir, ese que rescata formas de convivencia pasadas para insertarlas en la vida contemporánea ${ }^{9}$.

\section{Transformación de la lógica de la industria cultural (de lo masivo a lo local)}

La Industria Cultural, el concepto acuñado por Horkheimer y Adorno para referir el sistema de producción de sentido en las sociedades contemporáneas, se articula con base en una reducción del discurso que lo hace transitar de la razón hacia la emoción y del juicio crítico a la opinión. Tal es la lógica con la que operan la mercadotecnia y la publicidad. Ambas reducen al mínimo significante el impacto de un producto o servicio a fin de promover la identificación del mismo con un área de necesidad previamente construida. El gadget, los simuladores, las tecnologías escalables, la colección, las series dramáticas, la moda. Todos son sus productos. Los consumidores aparecemos en esta lógica como derivados funcionales que acoplamos los dispositivos a sus usos, las producciones a sus signos. Ello nos devuelve

\footnotetext{
${ }^{9}$ Hoy día, Illich podría resultar profético si consideramos el sentido que las ciudades comienzan a dar a formas de movilidad, convivencia y producción alternativos; modos más humanos y que tienen como fin promover formas de coexistencia más justas y "amigables" con el futuro (calles peatones, promoción del ciclismo y el transporte público, programas de autos compartidos, rescate y reconquista del Espacio Público, impulso a lo tradicional, habilitación de huertos urbanos, reforestación y programas de sustentabilidad barrial).
} 
una perspectiva distópica del hombre, una como la que anunció Palahniuk y que en 1998 se expuso a través de la película Fight Club, dirigida por David Fincher

..Man, I see in Fight Club the strongest and smartest men who have ever lived. I see all this potential, and I see it squandered. Goddammit, an entire generation pumping gas, waiting tables, slaves with white collars. Advertising has us chasing cars and clothes, working jobs we hate so we can buy shit we don't need. We're the middle children of history, man; no purpose or place. We have no Great War, no Great Depression. Our Great War is a spiritual war. Our Great Depression is our lives. We've all been raised by television to believe that one day we'd all be millionaires and movie gods and rock stars. But we won't; and we're slowly learning that fact. And we're very, very pissed off. (Chuck Palahniuk. Fight Club).

Y la gran guerra espiritual anunciada por el protagonista de la novela, Tyler Durden, el alter ego de un esclavo corporativo no es otra que la que se libra en contra el desarrollo, en contra de la competencia estúpida que nos hace matarnos unos a otros para alcanzar una meta que no existe más que en los plots televisivos, en las pantallas de los ordenadores y en las marquesinas de las grandes tiendas de ropa. La única batalla que puede llevarnos a triunfar es -según enseñanza de la misma obra- el progresivo ejercicio de la inmolación.

\section{Eventual transformación de la idea de desarrollo (lo local como efecto de la inmolación)}

El sacrificio, en el marco de muchas religiones, aparece como un mecanismo que habilita la comunicación con los dioses acercando, a quienes lo ofrecen, a una serie de dádivas por parte de aquellos. La lógica elemental de este ritual implica un "me das - te doy” y los participantes asumen que la obtención de un beneficio implica -a la vez- el deshacerse de algo; de algo querido o importante como podía ser una doncella, un guerrero o, en el mejor de los casos, un pichón o una cabra. El acto de desprendimiento se consideraba una inversión y por ello era importante.

La modernidad anuló la idea del sacrificio. En el mejor de los casos, la desacralizó sustituyéndola por la idea de heroísmo o inmolación: actos excepcionales que implicaban el 
riesgo personal para la obtención de un beneficio colectivo. Los sacrificios modernos se llevaron a cabo en el campo de batalla, en las relaciones entre gobiernos, en la salvación de empresas o en el descubrimiento de recursos. Todos ellos buscaron mantener un sistema, una forma de gobierno o un cierto ideal social.

Hoy, con la consolidación del mercado y el advenimiento de los llamados "Estados de Bienestar", la inmolación no es fácilmente concebible pues su significado se opone a la idea de avance o programa racional. Los héroes contemporáneos realizan proezas sacrificando su cotidianidad, capital y tiempos ocio para usarlos en el diseño de conocimientos, técnicas y

dispositivos que coadyuven a la consolidación dicho paradigma. La consecuencia de este avance aparece como un fenómeno irreversible que, en aras del consumo, succiona no solo el recurso humano sino de toda índole poniendo en riesgo al futuro de la sociedad, de la especie y del ecosistema.

El tiempo del productor se sacrifica en aras de la generación de bienes y servicios y ésta habilita la oferta. En el otro lado el consumidor deviene no solo en factor de legitimación sino en razón para la innovación y la generación de nuevas necesidades y el sostenimiento de la cadena productiva. Gracias al círculo vicioso de su actividad es que la relación trabajo - consumo - ocio se mantiene y hace girar las hélices que desplazan al barco del progreso. Por ello su rol, en la redefinición del consumo a través de la crítica, es fundamental. Será precisamente el consumidor el que tendrá que definir las inmolaciones venideras. El cúmulo de sacrificios que tendrán que hacerse en pro de una cierta garantía de subsistencia. Las víctimas tendrán que ser sus consumos y lo que éstos propicien será una menor acumulación, un menor desecho y una menor depredación de los recursos para producir satisfactores.

¿Qué es lo que se pide?

No tanto que el consumidor racionalice su consumo y sacrifique parcial y gradualmente la emotividad de las mercancías sino que adquiera una cierta perspectiva del carácter caduco del significado de las mismas. Filosóficamente implica adquirir la idea de que el sentido nace 
en la necesidad y no en la satisfacción de la misma por lo que el sacrificio del satisfactor podría ser necesario.

Our Great Depression is our lives. We've all been raised by television to believe that one day we'd all be millionaires and movie gods and rock stars. But we won't; and we're slowly learning that fact. And we're very, very pissed off. (Chuck Palahniuk).

La gran depresión que constituyen nuestras vidas es la solicitud que se hace por la inmolación. Deshacernos de las evidencias parciales y caducas de crecimiento o desarrollo y de todo ideal sublimado por las industrias culturales. Centrarnos de vuelta en la función y en el mantenimiento de ciertos niveles de socialidad que nos unan más a una especie de comunitarismo que a una sociedad global.

No se trata de una nueva forma de luddismo o una neo anarquía ecologista. El dispositivo, la conectividad, las comunicaciones y la información son recursos invaluables pero que, por lo mismo, demandan niveles de competencia más altos en lo que a su uso se refiere. El desafío de las comunicaciones en un futuro próximo implicaría formar una nueva clase de receptores para quienes la idea de necesidad fuese necesaria y que el sentido de los satisfactores al momento de consumir se ofrezca como directrices y no encubrimiento del nivel de implicaciones que tendrá al llenar un espacio vacío de sentido y al abrir otros. Para quienes la simplificación sea una ventaja y no un obstáculo al momento de enfrentar el imperativo desarrollista el consumo deberá perfilarse a la luz de un nuevo entendido sobre el avance social en un contexto de crisis (ecológica, de desperdicio, de espacio, tiempo, etc.); una idea para la que el desarrollo no constituya una entelequia sino una perspectiva clara e incluyente que se enuncie en plural. Desarrollos. 


\section{Referencias}

Baudrillard. J. “La sociedad de consumo”. España. Ed. Siglo XXI. (2011).

Baudrillard. J.” De la seducción. España.” Ed. Cátedra. (1981).

Baudrillard. J. “Cultura y simulacro”. España. Ed. Kairos. (1996).

Cassirer, E.” Antropología filosófica. México”. Ed. FCE - Breviarios. (2004).

Comité Invisible.” La insurrección que viene”. España. Ed. Melusina. (2009).

Eco, U. “Número Cero”. España. Ed. Bompiani. (2015).

Eco, U.” La estrategia de la ilusión. España”. Ed. Random House. (2012).

García Canclini N. “Consumidores y ciudadanos”. México. Ed. Grijalbo. (1995).

Garduño, G. Objeto de consumo y relato. De la narrativa de la necesidad a la necesidad de narrativas. Pre print UAEMex, (2015).

Horkheimer, M. y T. Adorno. La industria cultural. España. Ed. Trotta, (1994).

Ibañez, J. "Sociología de la vida cotidiana. España”. Siglo XXI, (2012).

Lindón, A. “La vida cotidiana y su espacio-temporalidad”. México. Ed. Anthropos. (2000).

Lobo, F. “Sentido común, simulación y paranoia”. España. Ed. Pepitas de Calabaza. (2015).

Palahniuk, C. “El club de la lucha”. España. Joaquín Mortíz. (2008).

Illich, I. Reflexiones sobre el concepto de contra-productividad, (s/f). Disponible en http://www.ivanillich.org.mx/5contrapro2.pdf 
Schwartzberg, H. La contraproductividad inherente de la economía de los servicios. Conversación entre Sajay, Samuel y Jean, Robert. Oakland, EU. 2002. Disponible en http://habitat.aq.upm.es/boletin/n26/ahsch.html 